\title{
Boundary Layer Receptivity to Localized Disturbances in Freestream Caused by a Vortex Ring Collision
}

\author{
Sh. Noro ${ }^{\dagger}$, Y. Suzuki, M. Shigeta, S. Izawa and Y. Fukunishi \\ Tohoku University, 6-6-01, Aramaki-Aoba, Sendai, 980-8579, Japan \\ $\uparrow$ Corresponding Author Email: noro@fluid.mech.tohoku.ac.jp
}

(Received December 27, 2011; accepted December 13, 2012)

\begin{abstract}
The receptivity of a smooth flat plate to localized disturbances in freestream is investigated experimentally and numerically. The disturbances are generated outside a nominally-zero-pressure-gradient laminar boundary layer by a collision of two identical vortex rings with opposite signs. The vortex rings are generated by intermittent ejections of short duration jets from nozzles facing each other in the spanwise direction. A pair of rolled up vortex rings is given as the initial disturbances in the direct numerical simulation, and the growth of a boundary layer is simulated for a range of the Reynolds number based on the displacement thickness of boundary layer, $704 \leqq \operatorname{Re} \square * \leqq 844$. In the experimental results, high- and low-speed regions aligned in the streamwise direction are observed in the boundary layer before the external disturbances in the freestream reach the outer-edge of the boundary layer. Although velocity fluctuations inside both regions become stronger downstream, a transition to turbulence takes place only in the highspeed region at approximately $\operatorname{Re} \square *=844$. In the numerical results, vortical fluctuations similar to the experiment appear near the wall immediately after the vortex-ring-type disturbances are added in the freestream, but it is found that the vortical fluctuations do not directly grow into strong vortical structures. On the contrary, the development of strong vortical structures that leads to transition is triggered by the external disturbances directly intruding the boundary layer.
\end{abstract}

Keywords: Receptivity, Boundary layer, Transition, Vortex ring.

\section{NOMENCLATURE}

\begin{tabular}{|c|c|}
\hline$U$ & freestream velocity \\
\hline$\delta^{*}$ & displacement thickness of boundary layer \\
\hline $\operatorname{Re}_{\delta^{*}}$ & $=U \delta^{*} / v:$ Reynolds number \\
\hline$\omega$ & vorticity \\
\hline$\gamma$ & blob strength \\
\hline$z$ & $\begin{array}{l}\text { streamwise, wall-normal and spanwise } \\
\text { coordinates }\end{array}$ \\
\hline
\end{tabular}

\section{INTRODUCTION}

Laminar-turbulent transition is an important problem in many wall-bounded flows of both scientific and engineering interests. The state of the boundary layer flow has a strong influence on the performance of turbomachinery and the drag of an airfoil. The transition depends on various conditions, not only a body shape but also environmental factors: freestream turbulence (FST), background acoustic noise, surface roughness, pressure gradient, and so on. Traditionally, the mainstream in the study of transition of a boundary layer has been the linear stability theory, in which the unstable modes are discussed by solving the linearized fourth-order ordinary differential equation, known as

$\begin{array}{ll}u & \text { velocity vector } \\ \bar{u} & \text { time-averaged component of } \mathrm{u} \\ u^{\prime} & \text { velocity fluctuation } \\ u_{r m s}^{\prime} & \text { rms of } \mathrm{u}^{\prime} \\ \hat{u} & \text { periodic components of } \mathrm{u}^{\prime} \\ \tilde{u} & \text { non-periodic components of } \mathrm{u}^{\prime}\end{array}$

the Orr-Sommerfeld equation. When the FST level is low, the theory is very useful to predict the eigen mode in a boundary layer at the early stage of the transition, such as the Tollmien-Schlichting (TS) waves on flatplate and the Görtler vortices at a concavely curved wall (Reed and Saric, 1989, Saric et al. 2003). The secondary three-dimensional instabilities become dominant in the downstream region where the amplitude of TS waves exceeds $1 \%$ of the freestream velocity. This type of transition is commonly referred as natural transition. If the FST level is high, the initial linear growth stage is bypassed and the transition occurs early. These two transition scenarios have been widely studied for various FST conditions and useful 
knowledge has been accumulated (Fransson et al. 2005).

The starting point of the transition process is not unique. Regardless of the type of the process, the transition process always starts from a receptivity process. Receptivity is a term used to describe the process by which ambient disturbances such as vorticity and acoustic sound in the freestream enter the boundary layer or the free shear layer and generate the instability waves inside. The seeds of disturbances are usually taken into a boundary layer at locations where it starts to grow, for instance, the leading edge of a flat plate and the attachment line of a body, because the receptivity becomes the highest at these locations except for roughness on the surface. Almost all studies on transition are based on the premise of this fact. Goldstein (1983) analytically studied the scale matching mechanism between long-wavelength FST and shorter wavelength TS waves using the method of matched asymptotic expansions for the unsteady boundary-layer equation near the leading edge and the Orr-Sommerfeld equation farther downstream. The effect of FST levels on boundary layer transition has also been discussed. For example, Schrader et al. (2010) focused on the relation between the vortical perturbations in the oncoming freestream and leading edge bluntness. They reported that the boundary layer is most sensitive to axial vorticity, triggering a streaky velocity fluctuation pattern, and vertical vorticity in the freestream is responsible for non-modal instability. The latter mechanism relies on the generation of streamwise vorticity through stretching and tilting of the vertical vortex column at the blunt leading edge. Nagarajan et al. (2007) investigated the effect of a blunt leading edge on the bypass transition, where the solution of homogeneous isotropic turbulence is imposed at the inflow boundary. For sharper leading edge and low FST, transition usually occurs through instabilities on low-speed streaks, whereas increasing either the FST level or leading edge bluntness brings another transition scenario in which the transition occurs through growth and breakdown of wavepacket like disturbances in the boundary layer. Saric et al. (2002) provides a detailed review on the boundary layer receptivity.

In this study, we focus on the receptivity of a smooth flat plate to localized disturbances in freestream above a boundary layer through the boundary layer edge. If the freestream includes local intense fluctuations, they might have direct influence on the boundary layer nearby. These direct effects of external disturbances on the boundary layer are still unclear. In our previous study, Shigeta et al. (2008) used a turbulencegenerating bar located outside a boundary layer, in order to generate a disturbance free of the leading edge receptivity. The bar was a hollow circular pipe with several holes for ejecting jets into its wake. The device generated a peakvalley structure near the wall. Besides that, velocity fluctuations consisted of low-frequency components below $50 \mathrm{~Hz}$ were observed near the wall before the outer disturbance directly reached the boundary layer. It appeared as if this low-frequency disturbance that jumped into the wall region of the boundary layer triggered the transition. The problem with the experiment was that the existence of the bar induced a pressure gradient on the wall, which might have influenced the transition process. Thus, in this study, the external disturbances are introduced by colliding two vortex rings, replacing the turbulencegenerating bar, and their effect on the growth of a boundary layer is discussed. Three-dimensional numerical simulation is also performed for better understanding of the phenomenon.

\section{EXPERIMENT AND NUMERICAL APPROACH}

\subsection{Experiment Setup}

The experiment is carried out in the blowout-type lowturbulence wind tunnel. The tunnel has a square test section of $500 \mathrm{~mm}$ by $500 \mathrm{~mm}$ with an adjustable ceiling to provide a zero pressure gradient along the streamwise direction. Figure 1(a) shows the schematic view of the experimental setup. All the experiments are conducted at the freestream velocity $U=5 \mathrm{~m} / \mathrm{s}$, where the ratio of the velocity fluctuation intensity to the freestream velocity urms $=\mathrm{U}$ is less than $0.25 \%$. The flat plate made of an aluminum alloy is horizontally mounted $200 \mathrm{~mm}$ away from the bottom floor of the test section. The size of the plate is $1800 \mathrm{~mm}$ long, 490 $\mathrm{mm}$ wide and $10 \mathrm{~mm}$ thick. An elliptical leading edge with a radius ratio $24: 1$ is attached to the plate. The joint is smoothly connected to avoid disturbances to enter the boundary layer. A flap of a chord length 190 $\mathrm{mm}$ is also attached to adjust the stagnation point at the leading edge so that no flow separation occurs at the leading edge of the upper surface. The pressure gradient on the plate is adjusted to be closer to zero as possible. The variation of pressure coefficient within the test section is less than 0.01 even when a disturbance generator is installed. The detail of the generator is explained later. The origin of the coordinate system is located at the center of the leading edge of the plate, where $\mathrm{x}, \mathrm{y}$ and $\mathrm{z}$ axes represent the streamwise, wallnormal and spanwise directions, respectively. The data is collected using hot-wire anemometry systems with a single probe and an X-probe, where the diameter and length of hot-wires are $5 \mu \mathrm{m}$ and $1 \mathrm{~mm}$, and the distance between the wires of the X-probe is $0.5 \mathrm{~mm}$. Third-order polynomial was used for both probe calibrations. For vorticity measurement, the data is obtained every $3 \mathrm{~mm}$ in $\mathrm{y}$ and $\mathrm{z}$ directions by rotating the $\mathrm{X}$-probe. The sampling frequency is $5 \mathrm{kHz}$ and a fourth-order Butterworthtype low-pass filter is used to cut-off fluctuations of frequency higher than $2.5 \mathrm{kHz}$. A Pentium IV CPU computer with the Linux operating system is used for the analog-to-digital (A/D) conversion and storage.

The disturbance generator used in this experiment consists of two equal units facing each other in the spanwise direction. Each unit has a loudspeaker (VISATON FRW5) and a funnel with a nozzle of $4 \mathrm{~mm}$ diameter to eject a short duration jet, as shown in Fig. 1(b). They are fixed on the sidewall of the test section at an angle of 20 degrees using a L-type cantilever, keeping the distance between the nozzle exits $120 \mathrm{~mm}$. The generator is installed $500 \mathrm{~mm}$ away from the leading edge $\left(\operatorname{Re}_{\delta}{ }^{*}=704\right)$ and $70 \mathrm{~mm}$ from the plate surface. The amplitude of operating signal to the loudspeaker is determined by a preliminary experiment 
without an ambient mean flow. Figure 2 shows the time variation of velocity measured at a point $80 \mathrm{~mm}$ right downstream of the nozzle exit, when one loudspeaker is driven by a square wave signal with a duty ratio 0.5 and a period of $\mathrm{T}=0.1 \mathrm{~s}$. The maximum voltage of the signal is approximately $10 \mathrm{~V}$. Strong peaks appear regularly every $0.1 \mathrm{~s}$ and the maximum velocity exceeds $10 \mathrm{~m} / \mathrm{s}$, whose value is 2.5 times larger than the freestream velocity. The jet is also visualized using a smoke wire and incense sticks. The result is shown in Fig. 3. Starting vortex ring is clearly observed in the transverse and cross sections.

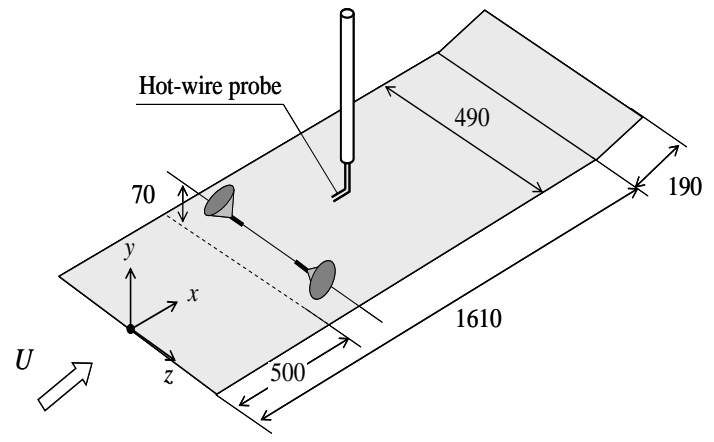

(a) experimental setup

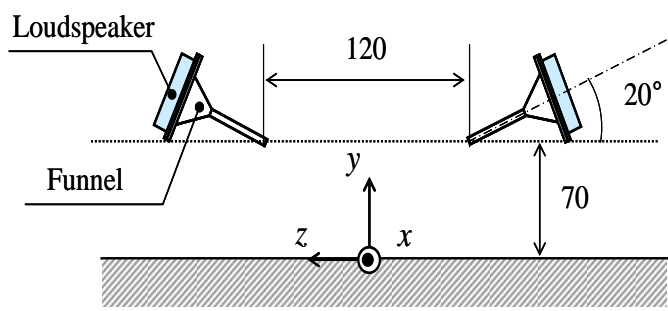

(b) disturbance generator

Fig. 1. Schematic view of experimental apparatus (dimension in $\mathrm{mm}$ ).

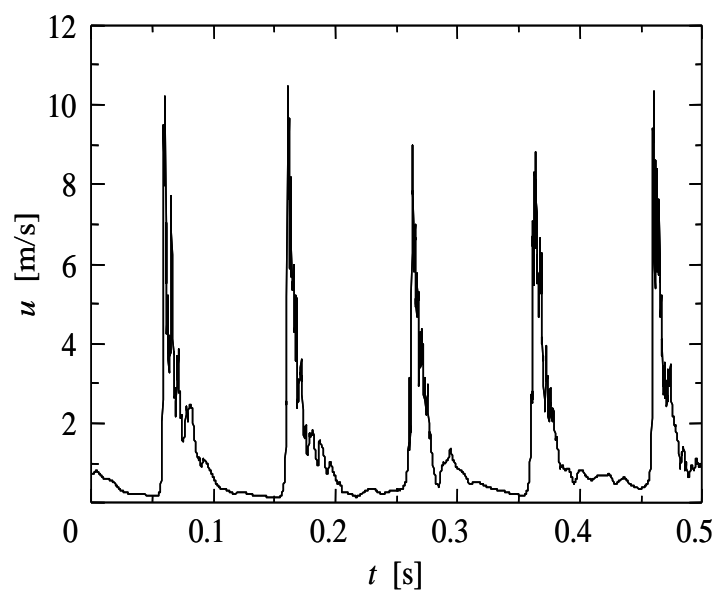

Fig. 2. Temporal variation of velocity measured $80 \mathrm{~mm}$ downstream from the nozzle exit.

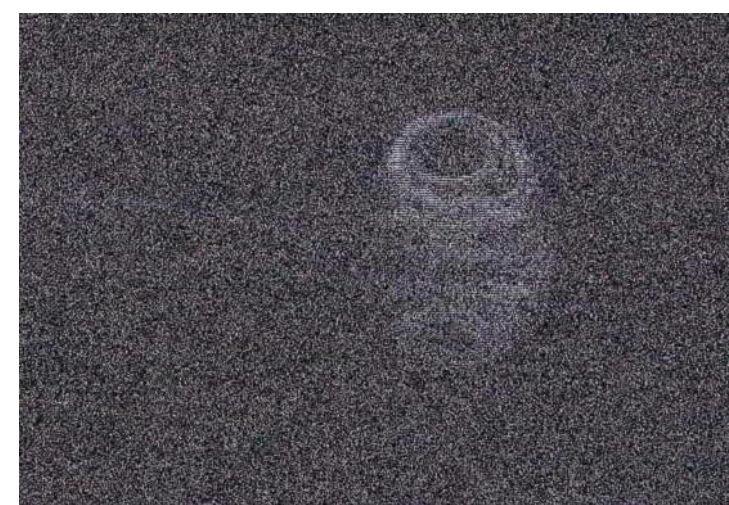

(a) transverse section

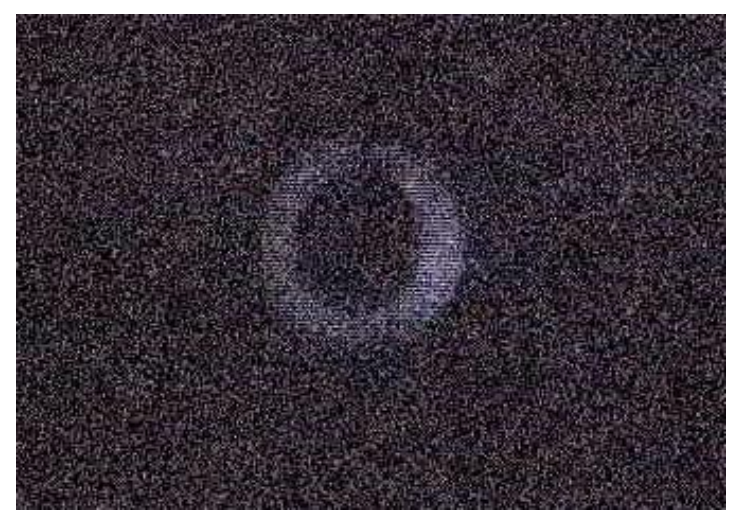

(b) cross section

Fig. 3. Vortex ring visualization generated by an intermittent jet ejecting into stationary fluid, using smoke wire for transverse section (a) and incense sticks for cross section (b).

In this experiment, two jets are ejected face to face in phase so that two equal vortex rings collide in the middle at regular intervals. Thus, the periodic component exists in the measured velocity signals in addition to the time-averaged component. In order to discuss the turbulence level inside a boundary layer, the time-averaged and the periodic components should be removed from the signal using a three-component decomposition,

$u(\mathbf{x}, t)=\bar{u}(\mathbf{x}, t)+\hat{u}(\mathbf{x}, t)+\tilde{u}(\mathbf{x}, t)$

where $\overline{\mathrm{u}}(\mathrm{x}, \mathrm{t})$ is the time-averaged component of the velocity, $\hat{\mathrm{u}}(\mathrm{x}, \mathrm{t})$ and $\tilde{\mathrm{u}}(\mathrm{x}, \mathrm{t})$ are the periodic and nonperiodic components of the velocity fluctuation. The turbulent intensity is defined as the root-meansquare (rms) value of the non-periodic component,

$\langle\tilde{u}(\mathbf{x}, t)\rangle=\sqrt{\frac{1}{N} \sum_{i=1}^{N} \tilde{u}(\mathbf{x}, t)}$

where the blankets indicate the ensemble-averaged value that is obtained using the operating signal of loudspeakers as a reference signal. Hereafter, $\langle\tilde{u}\rangle$ is referred to as the random component. 


\subsection{External Disturbances}

The characteristics of generated disturbances are investigated prior to discussing the receptivity of a smooth flat plate. Figure 4 shows the isosurface of the magnitude of the ensemble-averaged vorticity $|\omega|=\left(\omega_{\mathrm{x}}{ }^{2}\right.$ $\left.+\omega_{\mathrm{y}}{ }^{2}+\omega_{\mathrm{z}}{ }^{2}\right)^{1 / 2}$ at the different streamwise locations, $\mathrm{x}=$ $550 \mathrm{~mm}, 600 \mathrm{~mm}$ and $750 \mathrm{~mm}$. The experimental data were obtained by an X-type hotwire probe. The random component defined by Eq. (2) is only a few \% of the freestream velocity even in the mid-plane $\mathrm{z}=0 \mathrm{~mm}$ at $\mathrm{x}$ $=750 \mathrm{~mm}$, hence the data should be satisfying the Taylor's frozen eddy hypothesis and the temporal fluctuations recorded by the fixed hot-wire probe should accurately reflect the streamwise spatial fluctuations. At $x=550 \mathrm{~mm}, 50 \mathrm{~mm}$ downstream from the jet ejecting position, a pair of vortex rings is clearly observed outside the boundary layer at around $y=40 \sim$ $70 \mathrm{~mm}$. These vortex rings approach each other as they go downstream, owing to their induced velocities. The shape of vortex rings is an ellipse, not a circle shown in the smoke visualization (Fig. 3), because they are ejected into the cross flow (New et al. 2003, 2006). At $\mathrm{x}=750 \mathrm{~mm}$, they expand in the radial direction by collision and start collapsing.

\subsection{Simulation Method}

A direct numerical simulation is also performed to simulate the same flow. The computational domain is illustrated in Fig. 5. The domain covers the region: 500 $\mathrm{mm} \leqq \mathrm{x} \leqq 900 \mathrm{~mm}, 0 \mathrm{~mm} \leqq \mathrm{y} \leqq 80 \mathrm{~mm}$ and $-40 \mathrm{~mm} \leqq \mathrm{z}$ $\leqq 40 \mathrm{~mm}$, where the axes $\mathrm{x}, \mathrm{y}$ and $\mathrm{z}$ denote the streamwise, wall-normal and spanwise directions of the Cartesian coordinate system. The velocity components in these directions are $\mathrm{u}, \mathrm{v}$ and $\mathrm{w}$, respectively, and $\mathrm{p}$ is the pressure. The velocity profile of a Blasius boundary layer with the zero pressure-gradient is given as a base flow. The v-component of velocity is also provided to account the growth of the boundary layer.

non-dimensionalized differential equations for three perturbation velocity component,

$$
\frac{\partial u_{i}{ }^{\prime}}{\partial t}+u_{j} \frac{\partial u_{i}{ }^{\prime}}{\partial x_{j}}=-\frac{\partial p}{\partial x_{i}}+\frac{1}{\operatorname{Re}_{\delta^{*}}} \frac{\partial^{2} u_{i}{ }^{\prime}}{\partial x_{j} \partial x_{j}}
$$

are solved replacing the ordinary Navier-Stokes equations. The Reynolds number $\operatorname{Re}_{\delta^{*}}$ is defined based on the displacement thickness $\delta^{*}$ given by

$$
\delta^{*} 1.73 \sqrt{\frac{v x}{U}}
$$

The where $\mathrm{n}$ is kinematic viscosity. The value of $\delta^{*}$ at the inlet $x=500 \mathrm{~mm}$ is equal to $2.112 \mathrm{~mm}$. Thus, the inflow Reynolds number $\operatorname{Re}_{\delta^{*}}$ is 704 . These equations are discretized based on the multi-directional finite difference method (Kuwahara et al. 2003). For the convection terms, the third-order upwind scheme is used to stabilize the computation, while the secondorder central difference scheme is used for other terms. The second-order Crank-Nicolson implicit scheme is used for the time integration. The Poisson equation for the pressure is solved by the multigrid algorithm, where the grid spaces are uniform in the $\mathrm{x}$ and $\mathrm{z}$ directions, while in the $y$ direction, the grid points are concentrated near the wall. Total of $257 \times 129 \times 129$ grid points are used. The non-slip condition is applied along the plate surface, whereas the slip condition is imposed on the upper boundary that is defined as the streamline in freestream without disturbances. The Neumann condition is used as the pressure boundary condition.

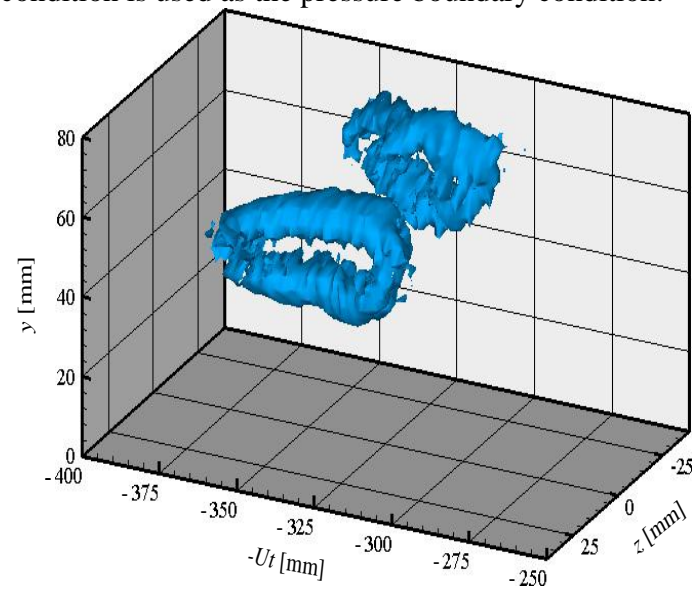

(a) $|\omega|=3001 / \mathrm{s}$ at $x=550 \mathrm{~mm}$

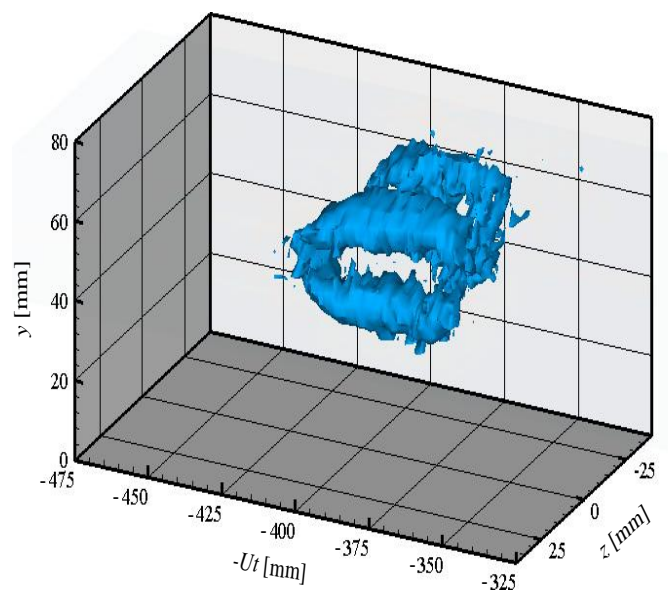

(b) $|\omega|=2301 / \mathrm{s}$ at $x=600 \mathrm{~mm}$

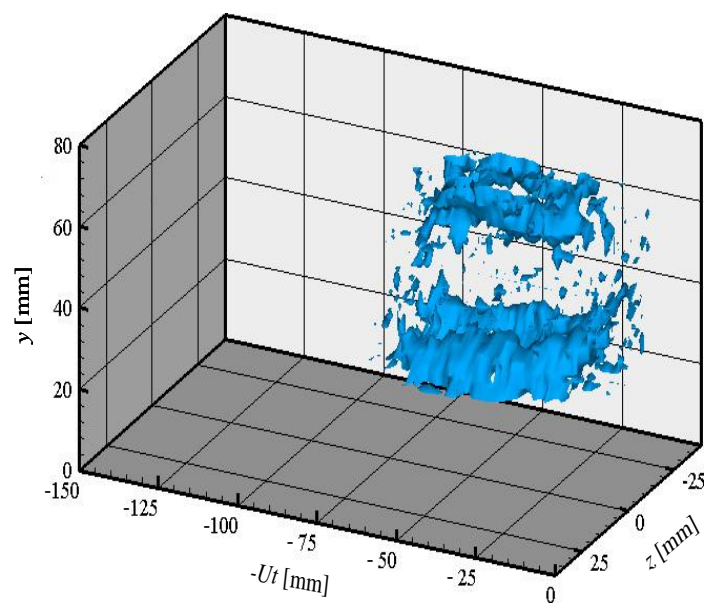

(c) $|\omega|=2301 / \mathrm{s}$ at $x=750 \mathrm{~mm}$

Fig. 4. Isosurface of vorticity magnitude $|\omega|$ at different streamwise locations. 


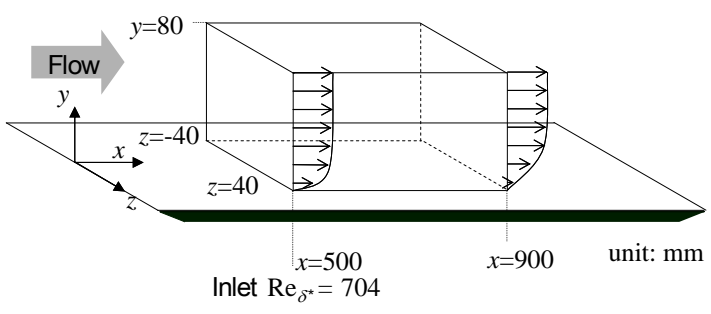

Fig. 5. Computational domain

In the computation, the velocity profile of the external disturbances visualized in Fig. 4 is simply modeled by the induced velocity profile of a pair of circular vortex rings. The shape of vortex rings is an ellipse, not a circle shown in Fig. 4, because they are ejected into the cross flow (New et al. 2003, 2006). At $\mathrm{x}=750 \mathrm{~mm}$, they expand in the radial direction by collision and start collapsing. First of all, the parameters of vortex ring such as circulation, radius and cross-section area are estimated from the experimental results. Table 1 shows the parameters used in the simulation. Each vortex is placed face to face at a distance of $20 \mathrm{~mm}(\mathrm{z}= \pm 10$ $\mathrm{mm})$ at $\mathrm{x}=550 \mathrm{~mm}$ and $\mathrm{y}=50 \mathrm{~mm}$. Then, they are represented by a group of vortex blobs and the induced velocity at each grid point is calculated from the BiotSavart law in the particle representation form, given by

$$
\mathbf{u}(\mathbf{x}, t)=-\sum_{\beta} \frac{1}{4 \pi\left|\mathbf{x}-\mathbf{x}_{\beta}(t)\right|^{3}}\left(\mathbf{x}-\mathbf{x}_{\beta}(t)\right) \times \gamma_{\beta}(t)
$$

where $\gamma_{\beta}(t)$ stands for the blob strength defined by the product between the blob vorticity $\omega_{\beta}$ and its volume (Winckelmans and Lenard, 1993). It should be noted that the mirror images are used to satisfy the slip-wall condition at $y=0 \mathrm{~mm}$. The obtained velocity profile is superimposed on the Blasius flow over a flat plate.

Table 1 Parameter of experimental results

\begin{tabular}{c|c|c}
\hline radius & circulation & cross-sectional area \\
\hline $10 \mathrm{~mm}$ & 0.123 & $55.9 \mathrm{~mm} 2$ \\
\hline
\end{tabular}

\section{RESUlts AND Discussions}

The following discussion is primarily described on the growth of perturbations inside a boundary layer generated by the localized external disturbances in the freestream, focusing on the random component of velocity fluctuation. Firstly, we discuss the response of a flat-plate boundary layer to the external disturbances based on the experimental data, and then, investigate the spatial development of velocity fluctuation nearwall region in detail by comparing the numerical result to that of the experiment.

\subsection{Experimental Results}

The direct effect of the disturbance generator itself and the route of leading edge receptivity followed by the growth inside the upstream boundary layer were checked. The profiles of streamwise velocity u obtained by ensemble averaging referencing the signal activating the generator for 30 times the data obtained at $\mathrm{z}=-40$, $0,40 \mathrm{~mm}$ and $\mathrm{x}=900 \mathrm{~mm}$, which is the most downstream location of the measurement region. The results are shown in Fig. 6. At each spanwise location, the velocity profile agrees well with the Blasius velocity profile. The $\mathrm{rms}$ profile of the velocity fluctuation u'rms at $\mathrm{z}=0 \mathrm{~mm}$ and $\mathrm{x}=410 \mathrm{~mm}$, which is the upstream point of the turbulence generator's location, also coincides with that without a generator.

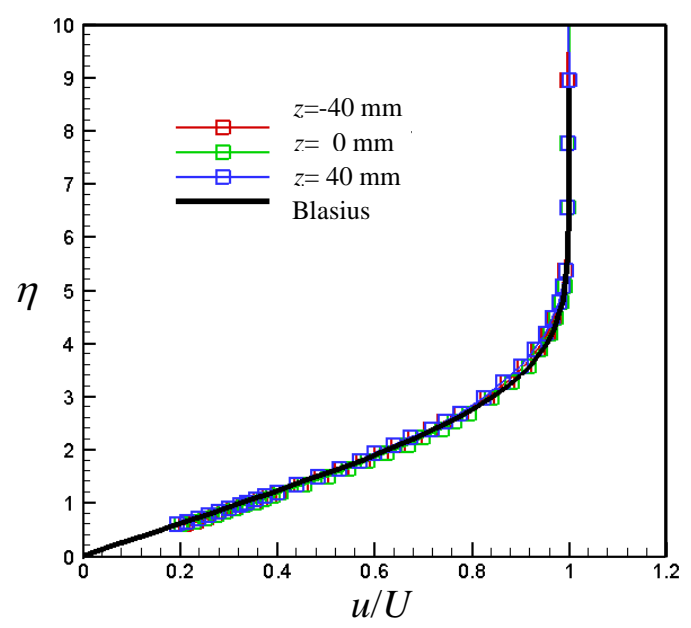

Fig. 6. Velocity profile $u=U$ at $x=900 \mathrm{~mm}$.

Figure 7 shows the contour maps of ensemble averaged velocity fluctuation $\hat{u}$ in $x y$ plane at $z=0 \mathrm{~mm}$. The positive and negative fluctuation patterns in the freestream become stronger as the vortex rings approach each other (Fig. 7-(b) and 7-(c)), and gradually weaken after they collapse (Fig. 7-(d) and 7(e)). In the present experiment, the same phenomena occur repeatedly since the jets are ejected intermittently at the constant interval of $0.1 \mathrm{~s}$. In Fig. 7(a), it is found that the negative fluctuation pattern is still left near the wall on the downstream side of the vortex rings. Thus, the effect of the previous ejection is not completely swept away from the flow field. As a result, the downstream positive velocity fluctuation induced by a pair of vortex rings above grows colliding to the anterior negative region. The location where the vortex rings reach the outer edge of the boundary layer is around $\mathrm{x}=700 \mathrm{~mm} \sim 750 \mathrm{~mm}$. The maximum value of the velocity fluctuation inside the boundary layer reaches about $4.5 \%$ of the freestream velocity in this $x y$ plane.

The profiles of ensemble-averaged velocity fluctuation $\hat{u}$ in zt plane at $y=3 \mathrm{~mm}$ are presented in Fig. 8. The positive and negative fluctuation regions align in the streamwise direction. By comparing with those in the xy plain shown in Fig. 7, it is found that the negative pattern on the downstream side is due to the effect of the previous vortex rings, while the positive pattern on the upstream side is due to the current vortex rings just above a boundary layer. They become gradually narrower and stronger around $\mathrm{z}=0 \mathrm{~mm}$ as they go downstream. The maximum value of the velocity fluctuation reaches $7 \%$ of the freestream velocity. Figure 9 shows the intensity of the random component $\tilde{u}$ in the same plane as Fig. 8. The value of ũ becomes stronger in the high speed region compared to the velocity fluctuation distributions. At the end of the measuring area $x=900 \mathrm{~mm}$, it reaches $15 \%$ of the freestream velocity. At the same time, the values of the 
spanwise and wall-normal random component $\tilde{v}$ and $\tilde{w}$ also increase to $9 \%$ and $7 \%$ of the freestream velocity. These results indicate that this region can be regarded as turbulent.

\subsection{Numerical Results}

Figure 10 presents the contour maps of streamwise velocity fluctuation $\mathrm{u}^{\prime}$ in $\mathrm{xy}$ plane at $\mathrm{z}=0 \mathrm{~mm}$. As well as the experiment, a pair of positive and negative fluctuations appears in the freestream because of the vortex collision. Besides, a positive fluctuation pattern inclined toward the streamwise direction is also observed in the vicinity of the wall at $t=37.0$. After the vortex rings reach the boundary layer at $\mathrm{t}=62.95$, this positive velocity region become elongated in the streamwise direction. This is clearly observed in the distribution of $\mathrm{u}^{\prime}$ in $\mathrm{yz}$ plane at $\mathrm{y}=3 \mathrm{~mm}$, shown in Fig. 11.

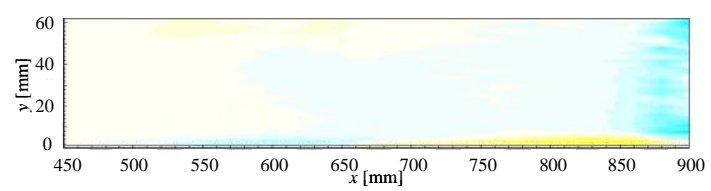

(a) $t / T=0$

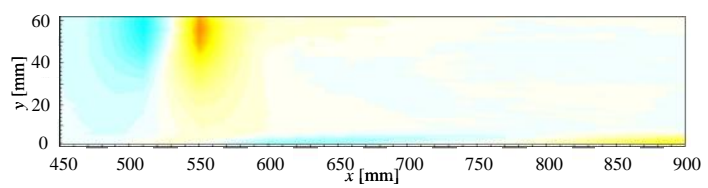

(b) $t / T=0.2$

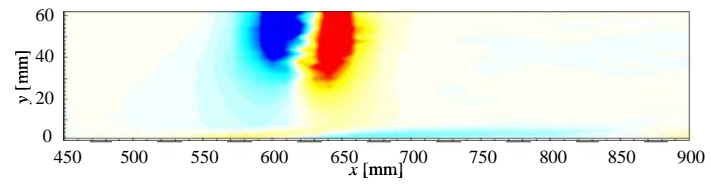

(c) $t / T=0.4$

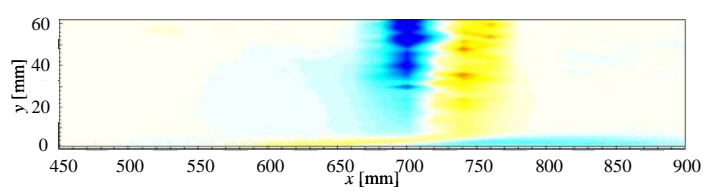

(d) $t / T=0.6$

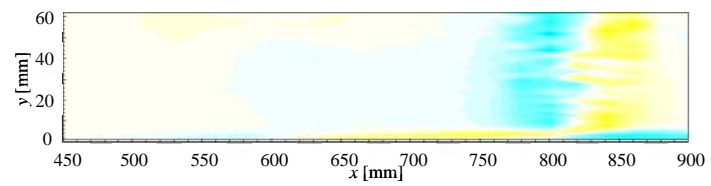

(e) $t / T=0.8$

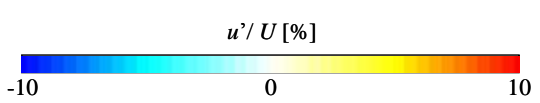

Fig. 7. Contour maps of ensemble-averaged velocity fluctuation $u^{\prime}=U$ in $x y$ plane.

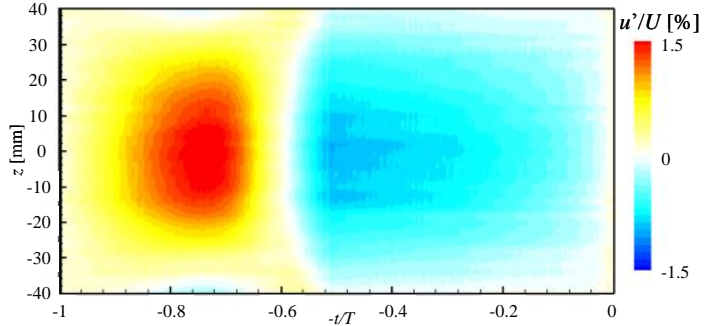

(a) $x=550 \mathrm{~mm}$

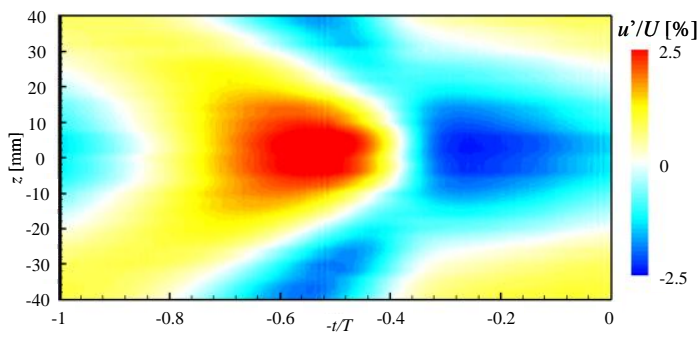

(b) $x=650 \mathrm{~mm}$

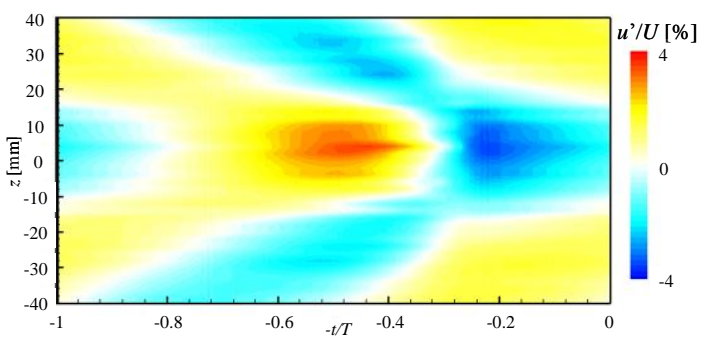

(c) $x=750 \mathrm{~mm}$

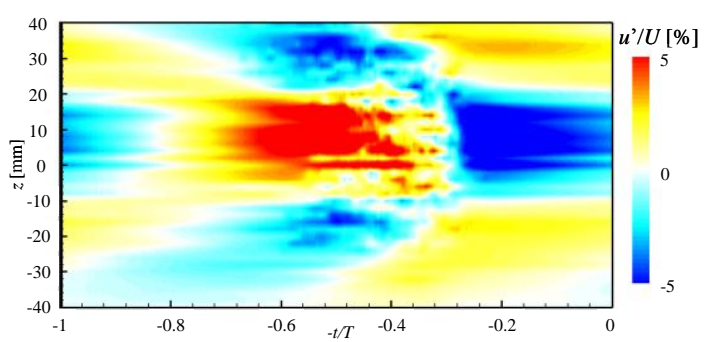

(d) $x=900 \mathrm{~mm}$

Fig. 8. Contour maps of ensemble-averaged velocity fluctuation $u=U$ in $z t$ plane at $y=3 \mathrm{~mm}$.

A high-speed region appears along the streamwise direction and is distributed from $\mathrm{x}=670 \mathrm{~mm}$ to 760 $\mathrm{mm}$. It accompanies low-speed regions on both spanwise sides, since the vortex rings approach each other flowing downstream. It should be noted that the collision of vortex rings is only a one-time event unlike the experiment; hence there is no negative region in its downstream side. 


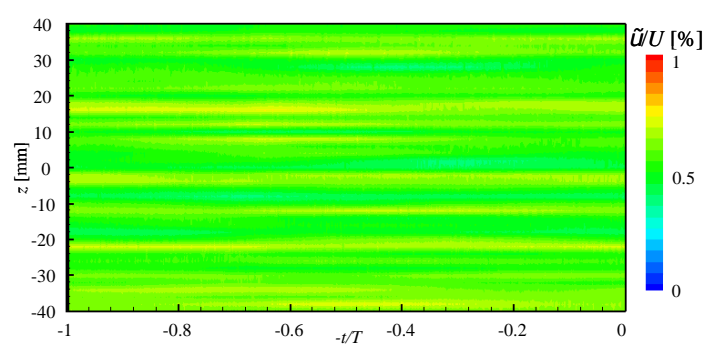

(a) $x=550 \mathrm{~mm}$

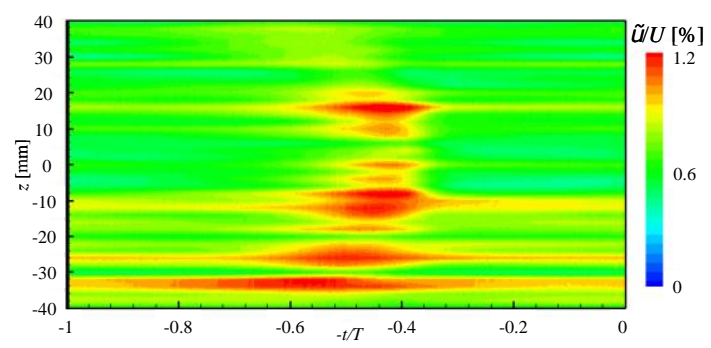

(b) $x=650 \mathrm{~mm}$

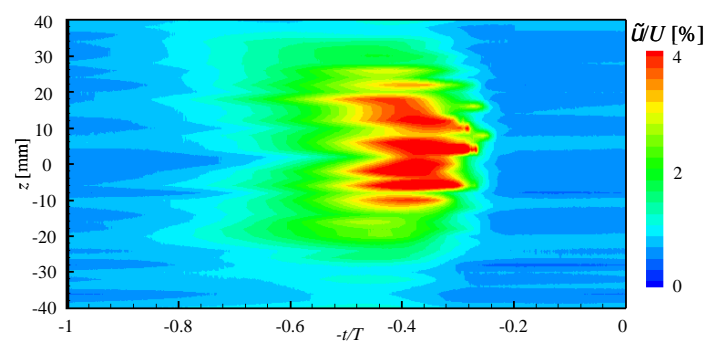

(c) $x=750 \mathrm{~mm}$

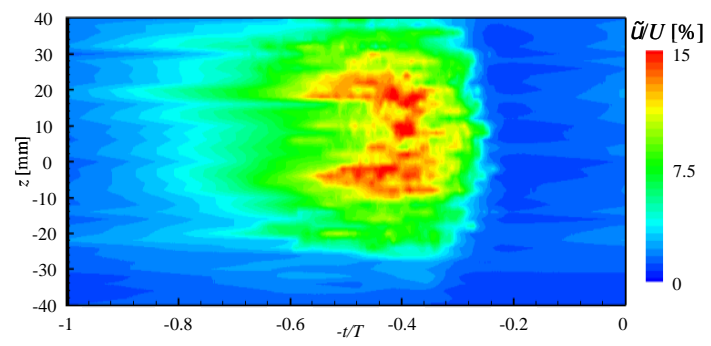

(d) $x=900 \mathrm{~mm}$

Fig. 9. Contour maps of random component of velocity fluctuation $\tilde{u}=U$ in $z t$ plane.

Figure 12 shows the growth of vortical structures visualized by the isosurface of $Q=0.0001$, where $Q$ is the second invariant of a velocity gradient tensor (Hunt et al. 1988). As well as the experiment, the collision makes the vortex rings enlarged in the radial direction. They intrude into the boundary layer at around $x=750$ $\mathrm{mm}$ and $\mathrm{t}=62.95$, and after that, they are mainly stretched in the streamwise direction owing to the effect of the wall. In the near wall region, small and large vortical structures appear once soon before the vortex rings approach the outer edge of the boundary layer. However, they become weaker afterward (Fig. 12-(b) and 12-(c)). After $\mathrm{t}=62.95$, several new vortical structures elongated along the streamwise directions are generated by the direct interaction with the vortex rings $((d) \sim(f))$. The streamwise velocity profiles in yz plane at $x=723 \mathrm{~mm}$ is shown in Fig. 13. In the figure, it is found that the velocity profiles are deformed around the colliding plane at $\mathrm{z}=0 \mathrm{~mm}$ and the velocity gradient becomes steeper in the vicinity of the wall. These numerical results suggest that the boundary layer transition observed in the experiment is due to the direct interaction between the external disturbances and the boundary layer, not due to the growth of the velocity fluctuations generated further upstream by the external disturbances in the freestream.

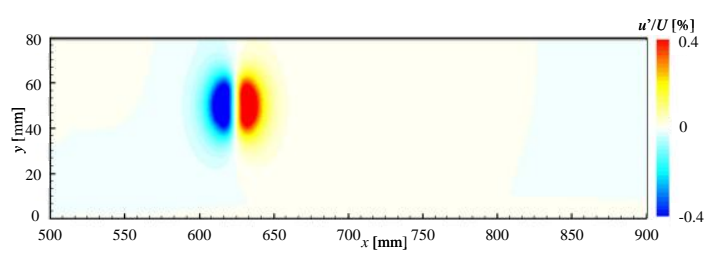

(a) $t=11.11$

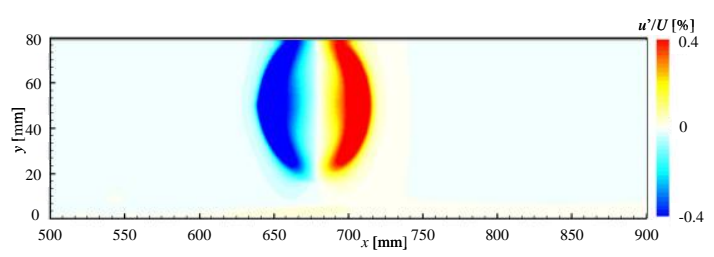

(b) $t=37.03$

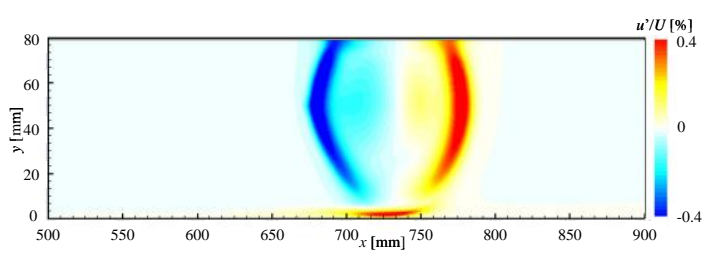

(c) $t=62.95$

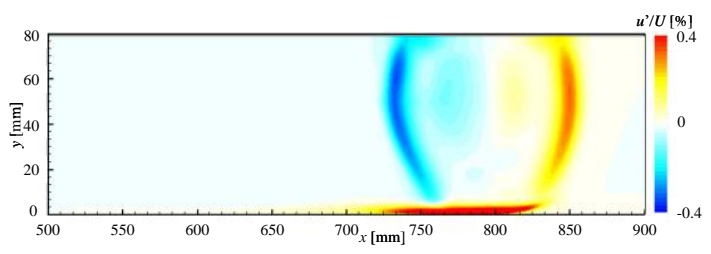

(d) $t=92.58$

Fig. 10. Contour maps of velocity fluctuation $u^{\prime} / U$ in $x y$ plane.

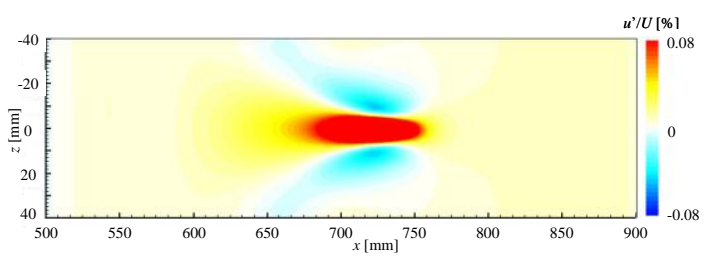

Fig. 11. Contour maps of velocity fluctuation $u^{\prime} / U$ in $x z$ plane at $t=62.95$. 


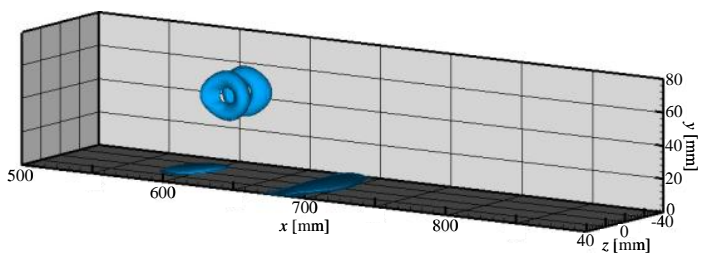

(a) $t=11.11$

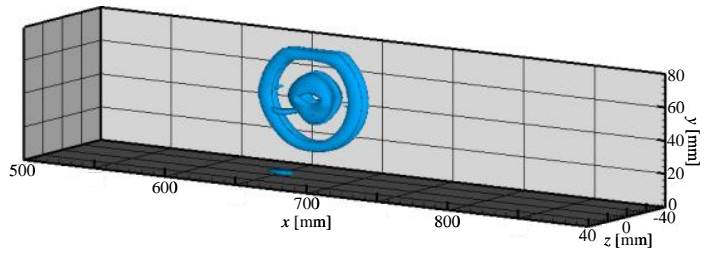

(b) $t=37.03$

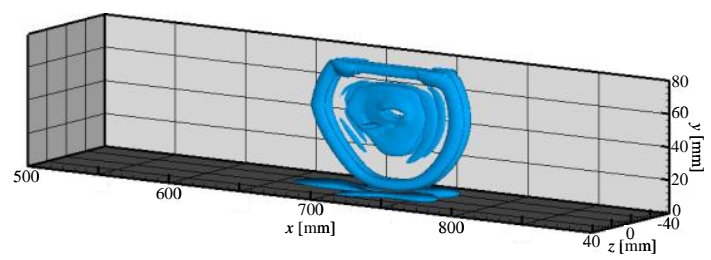

(c) $t=62.95$

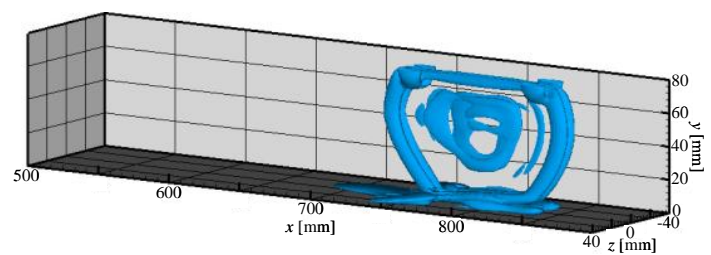

(d) $t=92.58$

Fig. 12. Isosurface of $Q=0.0001$.

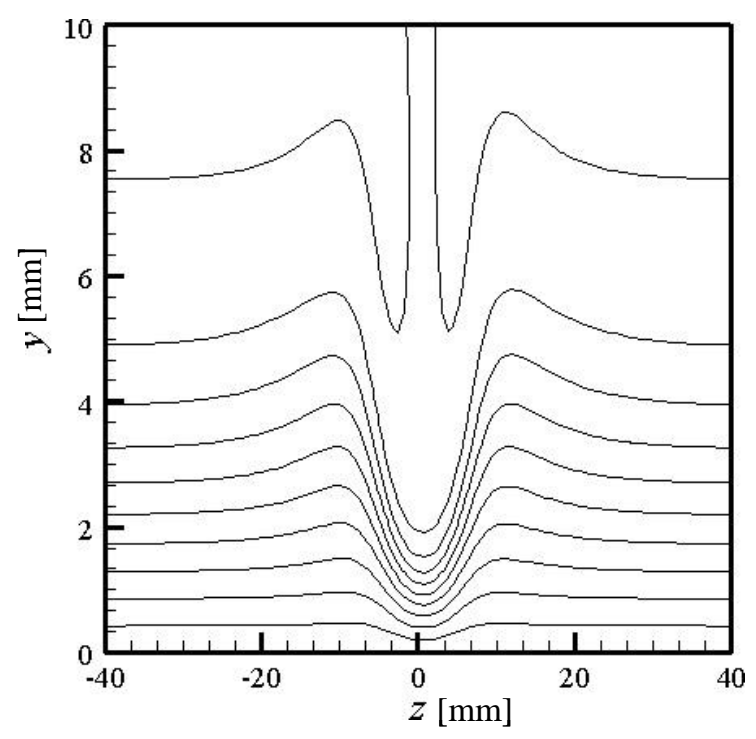

Fig. 13. Profiles of streamwise velocity $u / U$ in $x z$ plane at $x=723 \mathrm{~mm}$ and at $t=62.95$. The lines are drawn at a constant interval 0.1 .

\section{CONClusion}

Experimental and numerical studies were carried out to investigate the receptivity of a smooth flat plate to localized disturbances artificially introduced in freestream away from the leading edge. The disturbances were generated outside a nominallyzeropressure-gradient laminar boundary layer by the collision of two identical vortex rings with opposite signs. Owing to the collision of these vortex rings, a high-speed region was generated near the wall immediately below. The disturbances started to grow in this region and the boundary layer finally changed into a turbulent state. However, from the numerical results, it became clear that the boundary layer transition was triggered by the direct interaction with the external disturbances.

\section{REFERENCES}

Fransson, J.H.M., M. M. and P. Alfredsson (2005). Transition induced by free-stream turbulence. $J$. Fluid Mech. 527, 1-25.

Goldstein, M. (1983). The evolution of tollmienschlichting waves near a leading edge. $J$. Fluid Mech. 127, 59-81.

Hunt, J.C.R., W. A. and P. Moin (1988). Eddies, stream, and convergence zones in turbulent flows. Center for Turbulence Research Report CTR-S88, 193-208.

Kuwahara, K., K. S. and A. Bethancourt (2003). Incompressible flow simulation by using multidirectional finite difference scheme. numerical simulations of incompressible flows. In M. M. Hafez (Ed.), Numerical simulations of incompressible flows, 5 Toh Tuck Link, Singapore, pp. 311-326. World Scienticic.

Makita, H. and A. Nishizawa (1999). Deformation of internal structures caused by interaction between turbulent spots. T. JSME, Series B 64(632), 12831290.

Nagarajan, S., L. S. and J. Ferziger (2007). Leadingedge effects in bypass transition. J. Fluid Mech. 572, 471-504.

New, T.H., L. T. and S. Luo (2006). Effects of jet velocity profiles on a round jet in cross-flow. Experiments in Fluids 40, 859-875.

New, T.H., L. T. and S. Luo (2003). Elliptic jets in cross-flow. J. Fluid Mech. 494, 119-140.

Reed, H. and W. Saric (1989). Stability of threedimensional boundary layers. Ann. Rev. Fluid Mech. 21, 235-284. 
Sh. Noro et al. / JAFM, Vol. 6, No. 3, pp. 425-433, 2013.

Saric, W.S., R. H. and E. White (2003). Stability and transition of three-dimensional boundary layers. Ann. Rev. Fluid Mech. 35, 413-440.

Saric, W.S., R. H. and E. Kerschen (2002). Boundarylayer receptivity to free stream disturbances. Ann. Rev. Fluid Mech. 34, 291-319.

Schrader, L.-U., B. L. M. C. and D. Henningson (2010). Receptivity to free-stream vorticity of flow past a flat-plate with elliptic leading edge. J. Fluid Mech. 653, 245-271.
Shigeta, M., O. T. I. S. and Fukunishi (2008, August). The effect of outer disturbance on transition of a flat-plate boundary layer. In Proceedings of the 12th Asian Congress of Fluid Mechanics (12ACFM), Daejeon, Korea.

Winckelmans, G. and A. Lenard (1983). Contributions to vortex particle methods for the computation of three-dimensional incompressible unsteady flows. J. Comput. Phys. 109, 243-273. 